\title{
The High Current Ion Source System HOLCROSS
}

\author{
Nikolai R. Lobanov ${ }^{\dagger}$ \\ Moscow Engineering Physics Institute \\ Kashirskoye Chausse 31, Moscow 115409, Russian Federation
}

\begin{abstract}
The model HOLCROSS ion source is designed for the production of high current and brightness ion beams for application in particle accelerator injection and ion implantation. The cross type hollow cathode device in which the cross size of the cathode is greater than a longitudinal size is described. This system allows the creation of a quiet, cold and stable plasma of all cross sections. Compared with traditional hollow cathode systems this one is characterised by improved technical and operational performance. In the case of a duoplasmatron ion source the cross hollow cathode is optimised for gaseous charges such as noble gases, nitrogen, oxygen etc. A description of the source is given, together with some recent results. With a one aperture extraction system in which the square of the anode hole equals $0,25 \mathrm{~mm}^{2}$ this source will produce more than 15 $\mathrm{mA} \mathrm{H}_{1}{ }^{+}$at $8 \mathrm{keV}$ and $200 \mathrm{~mA}$ discharge current.
\end{abstract}

\section{INTRODUCTION}

In the field of ion implantation and particle accelerator's injection, there is a growing interest in ion beams of different mass with high brightness and high current. The project using an original hollow cathode ion source for a $\mathrm{MeV}$ - RF implanter has been developed at the Moscow Engineering Physics Institute [1]. Preliminary studies have been performed using only a cross-type hollow cathode device. This article summarises the investigations on a duoplasmatron ion source with cross-type hollow cathode (150-mm diam) performed in MEPhI.

\section{II.THE CROSS TYPE HOLLOW CATHODE ION SOURCE}

The experimental setup has already been described in a previous article [2],( see Figure 1). In brief, the hollow cathode is fabricated from a air-cooled cylindrical stainless steel chamber ( $150 \mathrm{~mm}$ diam, 0-30 $\mathrm{mm}$ depth). The cross size of the hollow cathode is greater than the longitudinal size. At the back of the cathode chamber, the operational gas is let in through a tube $4 \mathrm{~mm}$ in diameter and is pumped away through the anode aperture by a diffusion $1500 \mathrm{l} / \mathrm{s}$ oil pump. The square of the anode hole equals $0,25 \mathrm{~mm}^{2}$. Technical hydrogen was used.

† Correspondence should be send:Australian Institute of Physics, 1/21 Vale Street, North Melboume VIC 3052
The duoplasmatron ion source consists of few discharge chambers, followed by an small expansion cup.The expander ( $7 \mathrm{~mm}$ diam and $5 \mathrm{~mm}$ depth) with the extraction electrodeform the modified Pierce's system [2]. In the region between intermediate electrode and anode, an axial magnetic field, obtained by a permanent magnet, is applied. The magnetic flux lines are guided by ferromagnetic material. The influence of the magnetic field on the cathode region is negligible.

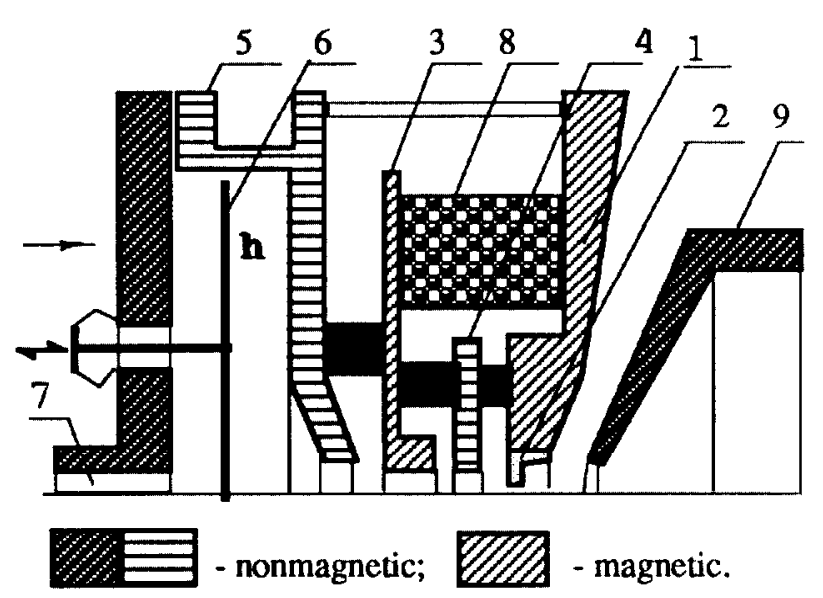

Figure 1. The experimental setup: 1- anode; 2 expander; 3- intermediate electrode; 4- additional electrode; 5 - cathode chamber; 6 - circle plate; 7-gas inlet; 8- magnets; 9- extractor.

An electronic system has been developed and built to run a hollow cathode and duoplasmatron ion source in dc mode. Cathode and anode are connected to the power supply. The cathode chamber is negatively biased with respect to the anode. The potentials of the intermediate and additional electrodes are floating.

All the measurements were performed with the source and extraction operated in dc mode. The ion optic system consists of an Einzel lens. The beam current was measured by a Faraday cup at a distance of $120 \mathrm{~cm}$ from the lens. The beam energy was varied between 5 and $15 \mathrm{keV}$.

\section{EXPERIMENTAL RESULTS}

In order to determine the optimal conditions for generating a plasma the effect of the discharge current $\left(I_{d}\right)$, discharge voltage $\left(\mathrm{U}_{\mathrm{d}}\right)$ and the source pressure $\left(\mathrm{P}_{\mathrm{a}}\right)$ on the distribution of the charged particles were investigated. In this experiment the intermediate and additional electrodes 
were pulled out. A cross hollow cathode discharge is easily established when the operating pressure is of the order of $10 \mathrm{~Pa}$. It is very stable discharge and transition into an arc discharge is not observed for the discharge current up to hundreds $\mathrm{mA}$ in dc mode.

Figure 2 shows the anode (discharge) voltage as function of the source pressure for a constant discharge current and a

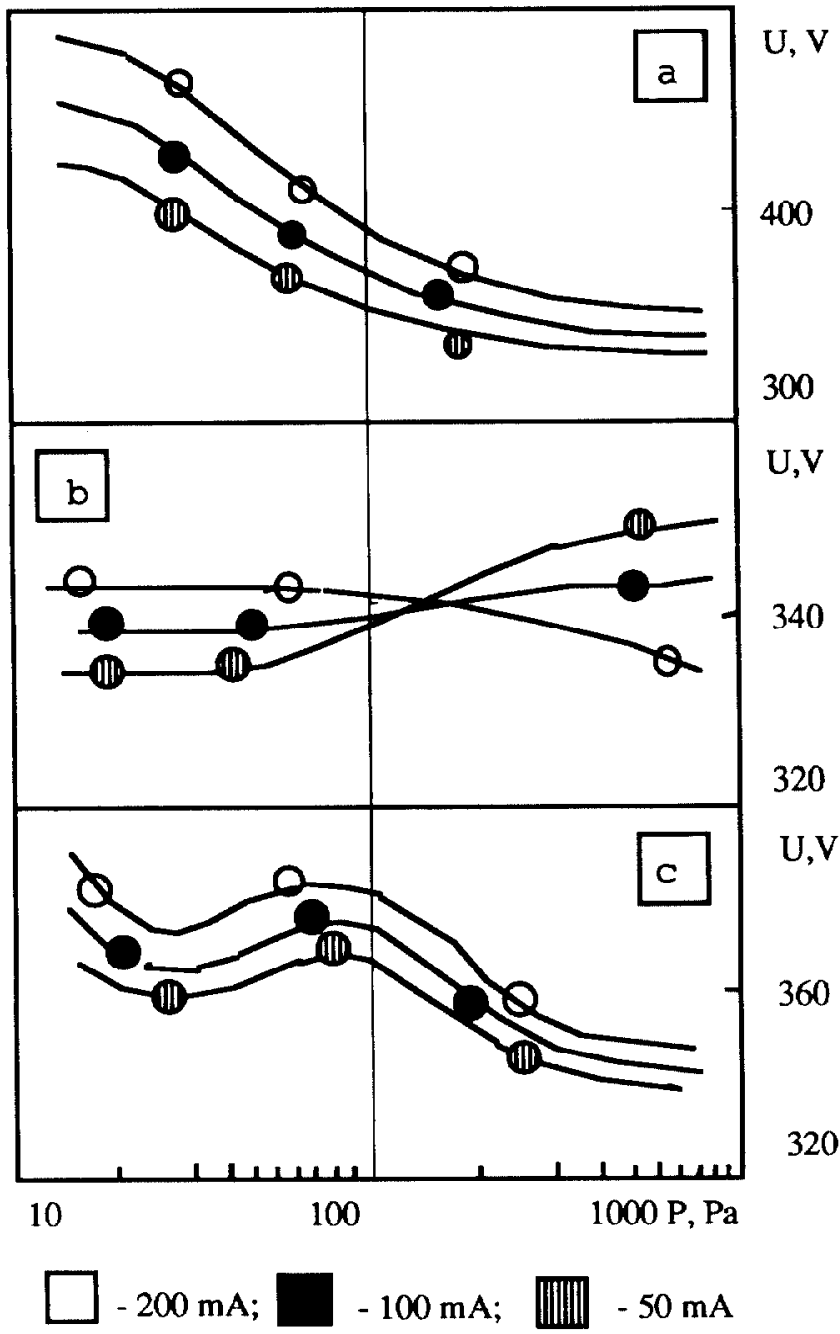

Figure 2. Dependence of the discharge voltage $U_{d}$ on the source pressure $\mathrm{P}_{\mathrm{a}}$ for a constant discharge current $\mathrm{I}_{\mathrm{d}}$ : a)- $h$ $=0 \mathrm{~mm} ; \mathrm{b})-h=6 \mathrm{~mm}$; $)-h=16 \mathrm{~mm}$.

cathode chamber depth $h$. It can be seen that for $h=0$ $\mathrm{mm}$ (fig. 2a) and for $h=16 \mathrm{~mm} ; \mathrm{P}_{\mathrm{a}}>50 \mathrm{~Pa}$ (fig. 2c) the curves repeat the same dependence for a glow discharge. The voltage dependency of the pressure on Figure $2 c$ is divided into two parts. For a pressure below $50 \mathrm{~Pa}$ and larger than $30 \mathrm{~Pa}$ the voltage is decreasing with the pressure decreasing. But for pressures below $30 \mathrm{~Pa}$ the situation is reversed. Now the voltage is rising and a glow discharge is transmitted into the high voltage ( electron beam ) mode.

Figure $2 \mathrm{~b}$ illustrates the relationship of the discharge voltage to the discharge current for another hollow cathode configuration. In this case, the hollow cathode's depth is 6 $\mathrm{mm}$. For a wide ranges of pressure and discharge current, the discharge voltage remains approximately constant.

The average discharge voltage $\langle\mathrm{U}\rangle$ on hollow cathode depth $h$ is plotted in Figure 3 for a constant source pressure. The results show that there is an optimal value of $h$ corresponding to the minimal discharge voltage. Voltage of $340 \mathrm{~V}$ can be obtained for a hollow cathode depth of about $6 \mathrm{~mm}$.

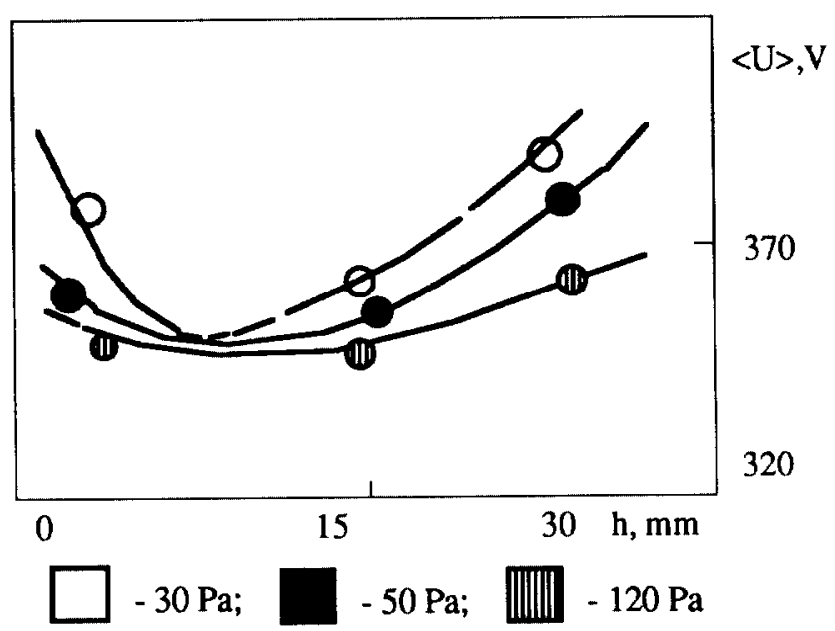

Figure 3. Dependence of the average discharge voltage $<\mathrm{U}>$ on the hollow cathode depth $h$ for a constant source pressure.

In the second part of the experiment, the visual observation of the discharge through the transparent cathode flange was conducted. A metal screen was installed instead of the circle plate. The results are shown in Figure $4 a-f$. One can see that the plasma occupation of the cathode chamber can be efficiently achieved due to the choice of combination of $\mathrm{I}_{\mathrm{d}}, \mathrm{Pa}_{\mathrm{a}}$ and $h$ values corresponding to the electron oscillation mode of the discharge.

The duoplasmatron type plasma generator is an example of use of the cross hollow cathode with the duoplasmatron ion source [2]. This system allows the creation of a quiet, cold and stable plasma of all cross sections. Compared with traditional hollow cathode systems this one is characterised by improved technical and operational performance. In the case of a duoplasmatron ion source the cross hollow cathode is optimised for gaseous charges such as noble gases, nitrogen, oxygen etc. With a one aperture extraction system this source will produce more than $15 \mathrm{~mA} \mathrm{H}_{1}{ }^{+}$at $8 \mathrm{keV}$ and $200 \mathrm{~mA}$ discharge current.For other monatomic 


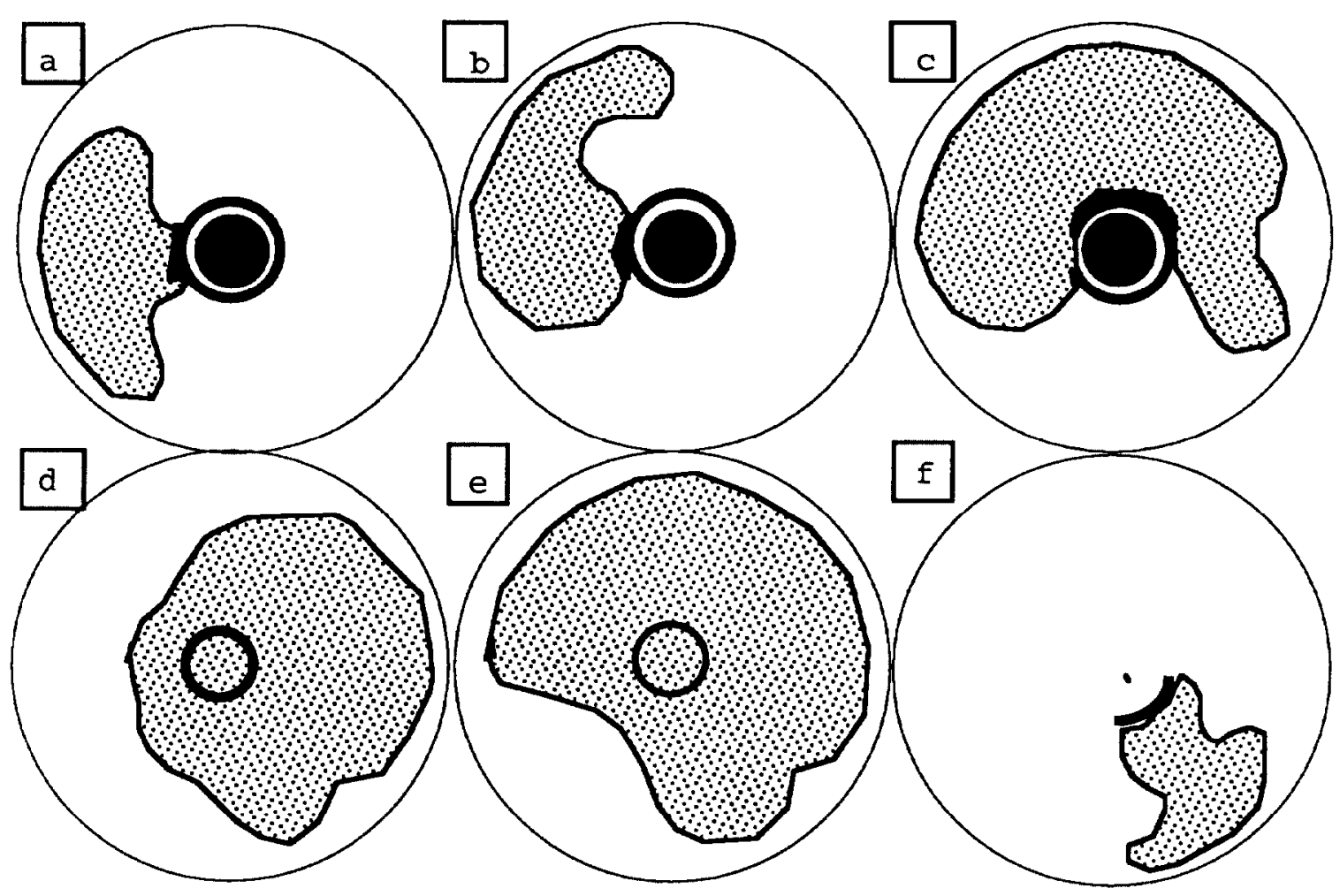

$\square$ - dark glow - faint glow - bright glow

Figure 4. Depiction of the plasma occupation of the cross hollow cathode's cavity for $h=6 \mathrm{~mm} ; \mathrm{I}_{\mathrm{d}}=100 \mathrm{~mA}$ ( for direction of vision see the arrow on fig. 1 ) : a- $P_{a}=10$ Pa, $U_{d}=360 \mathrm{~V} ; b-30,340 ; c-50,330 ; d-60,335 ; e-100,350$; f- 400,370

gases the beam currents scale as the inverse square root of the mass number $A$.

\section{CONCLUSION}

The cross hollow cathode system has certain advantages over the source of the previous article [2] of the same power dissipation, such as: high ionisation efficiency with low gas flow rates and good power efficiency. Due to the modular construction of the HOLCROSS system, it is fairly easy to introduce modifications. One special version uses an internal negatively biased sputtering target to produce ions from elemental metal samples. Another alternation is equipped with an additional cross type hollow cathode system placed between the auxiliary plasma generator and the extractor. A negative voltage is applied to the cathode so that dependent low pressure gas discharge is observed. All these advantages secure the application of the cross hollow cathode as an effective long-lived plasma generator.Further investigations of the cross hollow cathode system will be published.

\section{REFERENCES}

[1] N.R. Lobanov,"Plasma source of charge particles," Inventor's certificate N163467, Byull.Izobret. N9, (1991).

[2] A.A.Glazkov, N.R.Lobanov, V.T.Barchenko et.al.,"Duoplasmatron-type ion source with improved technical and operational performance for linear accelerator," Conference Record of the 1992 Third European Particle Accelerator Conference, Vol.2, pp.993-995. 